\title{
1 \\ VON NEUMANN ENTROPY AND RELATIVE POSITION BETWEEN SUBALGEBRAS
}

\author{
MARIE CHODA
}

Osaka Kyoiku University, Asahigaoka, Kashiwara 582-8582, Japan

marie@cc.osaka-kyoiku.ac.jp

\begin{abstract}
We give a numerical characterization of mutual orthogonality (that is, complementarity) for subalgebras. In order to give such a characterization for mutually orthogonal subalgebras $A$ and $B$ of the $k \times k$ matrix algebra $M_{k}(\mathbb{C})$, where $A$ and $B$ are isomorphic to some $M_{n}(\mathbb{C})(n \leq k)$, we consider a density matrix which is induced from the pair $\{A, B\}$. We show that $A$ and $B$ are mutually orthogonal if and only if the von Neumann entropy of the density matrix is the maximum value $2 \log n$, which is the logarithm of the dimension of the subfactors.
\end{abstract}

\section{INTRODUCTION}

There are several notions which describe some relative position between two subalgebras of operator algebras. As one of such notions between two subalgebras of finite von Neumann algebras, Popa introduced the notion of mutually orthogonal subalgebras (definition below) in [10. By the terminology complementarity, the same notion is investigated in the theory of quantum systems (see 8 for example).

The most primary interest would be the case where two subalgebras of some full matrix algebra, both of which are either maximal abelian or isomorphic to also some full matrix algebra. In such the cases, two subalgebras are connected by a unitary.

Our motivation for this work arises from the following fact:

In the previous paper [2], we defined a constant $h(A \mid B)$ for two subalgebras $A$ and $B$ of a finite von Neumann algebra, and showed the relative position between maximal abelian subalgebras $A$ and $B$ of $M_{n}(\mathbb{C})$ by using the values of $h(A \mid B)$. This $h(A \mid B)$ is a slight modification of Connes-Størmer relative entropy $H(A \mid B)$ in 4 (cf. [5). If $A_{1}$ and $A_{2}$ are maximal abelian subalgebras of $M_{n}(\mathbb{C})$, then there exists a unitary $u$ such that $A_{2}=u A_{1} u^{*}$, and then $h\left(A_{1} \mid A_{2}\right)$ coincides with the entropy $H(b(u))$ defined in [11] of the unistochastic matrix $b(u)$ induced by the unitary $u$. As a consequence, we showed that $A_{1}$ and $A_{2}$ are mutually orthogonal if and if $h\left(A_{1} \mid A_{2}\right)=H(b(u))=\log n$. This means that $A_{1}$ and $A_{2}$ are mutually orthogonal if and if $h\left(A_{1} \mid A_{2}\right)$ is maximal and equals to the logarithm of the dimension of the subalgebras. Related results in the case of subfactors of the type $\mathrm{II}_{1}$ factors are obtained in [3]. Here, it does not hold in general that $H\left(A_{1} \mid A_{2}\right)=H(b(u)$ ) (see, for example [9, Appendix] ).

On the other hand, Petz showed in [8] for subalgebras $A$ and $B$ of $M_{n}(\mathbb{C})$ that if $A$ is homogeneous and abelian, then $H(A \mid B)$ is maximal if and only if $A$ and

\footnotetext{
${ }^{1}{ }_{2010}$ Mathematics Subject Classification : 46L55; 46L37, 46L40

Key words and phrases. entropy, unitary, density matrix .

The author was supported in part by JSPS Grant No.20540209.
} 
$B$ are complementary. Here homogeneous means that all minimal projections of $A$ have the same trace. Also he remarked that Connes-Størmer relative entropy cannot characterize the complementarity of subalgebras in the general case.

In this paper, we study the case when the subalgebras $A$ and $B$ in question are isomorphic to some $M_{n}(\mathbb{C})$. We introduce some density matrix arising from the pair $\{A, B\}$, and we show that the von Neumann entropy of the density matrix gives a characterization of the mutual orthogonality (that is the complementarity).

In order to define the entropy for automorphisms of operator algebras, two kind of notion of a finite partition of unity played an important role. One was used by Connes-Størmer, and it corresponds to a finite measurable partition of a given space in the ergodic theory (see [5] [6] for example). The other was used by Alicci and Fannes in [1] and it is called a finite operational partition of unity. Here, we apply the latter, that is operational partition of unity, and we give a numerical characterization for pairs of mutually orthogonal subalgebras which are both isomorphic to some full matrix algebra of the same size.

The paper is organized as follows. After preliminaries on basic notions in Section 2 , in Section 3 we define some density matrix which is closely related to subfactors $A$ and $B$ which are both isomorphic to some $M_{n}(\mathbb{C})$, and we show that $A$ and $B$ are mutually orthogonal if and only if the von Neumann entropy of the density matrix is the maximum value $2 \log n$, which is the $\operatorname{logarithm}$ of the dimension of the subfactors.

\section{Preliminaries}

Here we summarize notations, terminologies and basic facts.

Let $M$ be a finite von Neumann algebra acting on a separable Hilbert space, and $\tau$ be a fixed normal faithful tracial state. In the case where $M$ is the algebra $M_{n}(\mathbb{C})$ of $n \times n$ matrices, $\tau(x)=\operatorname{Tr}(x) / n$, where $\operatorname{Tr}$ the usual standard trace on $M_{n}(\mathbb{C})$. The norm $\|x\|_{\tau}$ is given by $\|x\|_{\tau}=\tau\left(x^{*} x\right)^{1 / 2}$ for all $x \in M$. By a von Neumann subalgebra $A$ of $M$, we mean that $A$ is a $*$-subalgebra closed in the weak operator topology, the unit of which is the same with the unit of $M$. A conditional expectation of $M$ onto a von Neumann subalgebra $A$ of $M$ is a completely positive linear map $E_{A}: M \rightarrow A$ with $E_{A}(a x b)=a E_{A}(x) b$ for all $x \in M$ and $a, b \in A$. In the case of a finite von Neumann algebra $M$ with a faithful normal tracial state $\tau$, there exists always a unique faithful normal conditional expectation $E_{A}$ of $M$ onto a von Neumann subalgebra $A$ of $M$ such that $\tau(x a)=\tau\left(E_{A}(x) a\right)$ for all $x \in M$ and $a \in A$. It is called the conditional expectation with respect to $\tau$.

2.1. Mutually orthogonal (or complementary) subalgebras. Let $A$ and $B$ be von Neumann subalgebras of $M$. In [10, Lemma 2.1], Popa showed that the following conditions are equivalent.

(1) $\tau(a b)=0$ for $a \in A, b \in B$ with $\tau(a)=\tau(b)=0$;

(2) $\tau(a b)=\tau(a) \tau(b)$ for all $a \in A, b \in B$;

(3) $\|a b\|_{\tau}=\|a\|_{\tau}\|b\|_{\tau}$ for all $a \in A, b \in B$;

(4) $E_{A} E_{B}(x)=\tau(x) 1_{M}$, for all $x \in M$;

(5) $E_{A}(B) \subset \mathbb{C} 1_{M}$.

Moreover (1) - (5) are equivalent with the analogue conditions obtained by interchanging $A$ with $B$. 
Two von Neumann subalgebras $A$ and $B$ of $M$ are called mutually orthogonal if one of the above conditions (1) - (5) is satisfied ([10, Definition 2.2]).

Mutually orthogonal subalgebras are also called complementary subalgebras, (see, 7] 8] for example).

2.2. Density matrix and von Neumann entropy. By a density matrix, we mean a positive semidefinite matrix $\rho$ such that $\operatorname{Tr}(\rho)=1$. To a density matrix $\rho$, the von Neumann entropy $S(\rho)$ is given by $S(\rho)=\operatorname{Tr}(\eta(\rho))$. Here, $\eta$ is defined on the interval $[0,1]$ by

$$
\eta(t)=-t \log t \quad(0<t \leq 1) \quad \text { and } \quad \eta(0)=0 .
$$

\section{MAin RESUlts}

Let $M_{n}(\mathbb{C})$ be the algebra of $n \times n$ complex matrices, and let $T r$ be the trace of $M_{n}(\mathbb{C})$ with $\operatorname{Tr}(p)=1$ for every minimal projection $p$. Let $L$ be a finite von Neumann algebra, and let $\tau_{L}$ be a fixed normal faithful tracial state.

We let $M=M_{n}(\mathbb{C}) \otimes L$, and let $\tau_{M}=\operatorname{Tr} / n \otimes \tau_{L}$.

3.1. We consider the subalgebra $N=M_{n}(\mathbb{C}) \otimes 1_{L}$ of $M$. In this case, the conditional expectation $E_{N}$ with respect to $\tau_{M}$ satisfies that

$$
E_{N}(x \otimes y)=\tau_{L}(y) x \otimes 1_{L}, \quad x \in M_{n}(\mathbb{C}), \quad y \in L .
$$

The following lemma is an easy consequence from the definition, and it is essential to our study.

3.1.1. Lemma. Let $N=M_{n}(\mathbb{C}) \otimes 1_{L}$ and let $u \in M$ be a unitary operator. Then $N$ and $u N u^{*}$ are mutually orthogonal if and only if

$$
E_{N}\left(u^{*}\left(a \otimes 1_{L}\right) u\right)=\tau_{M}\left(a \otimes 1_{L}\right) 1_{M}=\frac{\operatorname{Tr}(a)}{n} 1_{M}, \quad \text { for all } \quad a \in M_{n}(\mathbb{C}) .
$$

Proof. Assume that $N$ and $u N u^{*}$ are mutually orthogonal, that is,

$$
E_{N} E_{u N u^{*}}(x)=E_{u N u^{*}} E_{N}(x)=\tau_{M}(x) 1_{M}, \quad \text { for all } \quad x \in M .
$$

Then $u E_{N}\left(u^{*} x u\right) u^{*}=E_{u N u^{*}}\left(E_{N}(x)\right)=\tau_{M}(x) 1_{M}$, for all $x \in N$. This implies that

$$
E_{N}\left(u^{*} x u\right)=\tau_{M}(x) 1_{M}, \quad \text { for all } x \in N .
$$

Conversely, assume that $E_{N}\left(u^{*} x u\right)=\tau_{M}(x) 1_{M}$, for all $x \in N$. Then

$$
E_{u N u^{*}}(x)=u E_{N}\left(u^{*} x u\right) u^{*}=\tau_{M}(x) 1_{M}
$$

for all $x \in N$. Hence

$$
E_{u N u^{*}} E_{N}(x)=\tau_{M}(x) 1_{M} \quad \text { for } \text { all } x \in M
$$

so that $N$ and $u N u^{*}$ are mutually orthogonal. 
3.2. Let $\left\{e_{i j} ; i, j=1, \cdots, n\right\}$ be a system of matrix units of $M_{n}(\mathbb{C})$, so that

$$
e_{i j}^{*}=e_{j i}, \quad e_{i j} e_{s t}=\delta_{j s} e_{i t}, \quad \sum_{i=1}^{n} e_{i i}=1_{M_{n}(\mathbb{C})} .
$$

Then each $x$ in $M=M_{n}(\mathbb{C}) \otimes L$ is written in the unique form:

$$
x=\sum_{i, j=1}^{n} e_{i j} \otimes x_{i j}, \quad x_{i j} \in L,
$$

and $u=\sum_{i, j=1}^{n} e_{i j} \otimes u_{i j}$ is a unitary in $M$ if and only if

$$
\sum_{j=1}^{n} u_{i j} u_{k j}^{*}=\delta_{i k} 1_{L} \quad \text { and } \quad \sum_{i=1}^{n} u_{i j}^{*} u_{i k}=\delta_{j k} 1_{L} .
$$

We give a characterization for a unitary $u \in M$ to satisfy that $N$ and $u N u^{*}$ are mutually orthogonal.

3.2.1. Theorem. Assume that a von Neumann subalgebra $N$ of $M$ is given by $N=M_{n}(\mathbb{C}) \otimes 1_{L}$ and let $u \in M$ be unitary. Then $N$ and $u N u^{*}$ are mutually orthogonal if and only if

$$
\tau_{L}\left(u_{i j}^{*} u_{k l}\right)=\delta_{i k} \delta_{j l} \frac{1}{n}, \quad \text { for all } \quad i, j, k, l=1, \cdots, n .
$$

Proof. Assume that $N$ and $u N u^{*}$ are mutually orthogonal. Then by Lemma 3.1.1

$$
E_{N}\left(u^{*}\left(e_{i j} \otimes 1_{L}\right) u\right)=\delta_{i j} \frac{1}{n} 1_{M} .
$$

On the other hand, since

$$
u^{*}\left(e_{i j} \otimes 1_{L}\right) u=\sum_{l, t=1}^{n} e_{l t} \otimes u_{i l}^{*} u_{j t}, \text { for all } i, j=1, \cdots, n,
$$

by applying that $E_{N}(x \otimes y)=\tau_{L}(y) x \otimes 1_{L}$, we have that

$$
E_{N}\left(u^{*}\left(e_{i j} \otimes 1_{L}\right) u\right)=\sum_{l, t=1}^{n} \tau_{L}\left(u_{i l}^{*} u_{j t}\right) e_{l t} \otimes 1_{L} .
$$

Hence $\sum_{l, t=1}^{n} \tau_{L}\left(u_{i l}^{*} u_{j t}\right) e_{l t}=\delta_{i j} \frac{1}{n} 1_{M_{n}(\mathbb{C})}$. This means that

$$
\tau_{L}\left(u_{i j}^{*} u_{k l}\right)=\delta_{i k} \delta_{j l} \frac{1}{n} \quad \text { for all } i, j, k, l=1, \cdots, n .
$$

Conversely, assume that $\tau_{L}\left(u_{i j}^{*} u_{k l}\right)=\delta_{i k} \delta_{j l} \frac{1}{n}$ for all $i, j, k, l=1, \cdots, n$. Then we have that

$$
E_{N}\left(u^{*}\left(e_{i j} \otimes 1_{L}\right) u\right)=\sum_{l, t=1}^{n} e_{l t} \otimes \tau_{L}\left(u_{i l}^{*} u_{j t}\right) 1_{L}=\sum_{l=1}^{n} e_{l l} \otimes \delta_{i j} \frac{1}{n} 1_{L}=\delta_{i j} \frac{1}{n} 1_{M}
$$

for all $i, j, k, l=1, \cdots, n$. Hence $N$ and $u N u^{*}$ are mutually orthogonal, by Lemma 3.1.1. 
3.2.2. Note. Theorem 3.2.1 implies that if $N=M_{n}(\mathbb{C}) \otimes 1_{L}$ and if $N$ and $u N u^{*}$ are mutually orthogonal for some unitary $u \in M=M_{n}(\mathbb{C}) \otimes L$, then the set $\left\{u_{i j} / \sqrt{n} ; i, j=1, \cdots, n\right\} \subset L$ has to be an orthonormal system with respect to the inner product induced by $\tau_{L}$ so that $\operatorname{dim}(L) \geq n^{2}$.

3.3. Entropy associated to an inner conjugate pair of subfactors. In order to give a numerical characterization for mutually orthogonal subalgebras which are all isomorphic to $M_{n}(\mathbb{C})$, we apply the notion of a finite operational partition $X$ of unity of size $k$ and the density matrix $\rho_{\phi}[X]$ which were introduced by Alicki and Fannes in [1].

3.3.1. Finite operational partition. Let $A$ be a unital $C^{*}$-algebra. A finite operational partition of unity of size $k$ is a set $X=\left\{x_{1}, \ldots, x_{k}\right\}$ of elements of $A$ satisfying

$$
\sum_{i}^{k} x_{i}^{*} x_{i}=1_{A}
$$

We remark that a similar terminology "finite partition" is usually used in the different following form: A finite subset $\left\{x_{1}, \ldots, x_{k}\right\}$ in $A$ is called a finite partition of unity if they are nonnegative operators in $A$ such that $1_{A}=\sum_{i=1}^{n} x_{i}$. See [5] or [6].

3.3.2. Density matrix $\rho[X]$. Let $\phi$ be a state of $A$. To a finite operational partition $X$ of unity of size $k$, we associate a $k \times k$ density matrix $\rho_{\phi}[X]$ such that the $(i, j)$-coefficient $\rho_{\phi}[X](i, j)$ of $\rho_{\phi}[X]$ is given by

$$
\rho_{\phi}[X](i, j)=\phi\left(x_{j}^{*} x_{i}\right), \quad i, j=1, \cdots, k .
$$

In the case that $A$ is a finite von Neumann algebra and that $\phi$ is a given tracial state $\tau$ of $A$, then we denote $\rho_{\tau}[X]$ simply by $\rho[X]$.

3.3.3. Finite operational partition induced by a unitary $u$. Now let $M_{n}(\mathbb{C})$ be the algebra of $n \times n$ complex matrices and let $\operatorname{Tr}$ be the trace with $\operatorname{Tr}(p)=1$ for every minimal projection $p$. Let $L$ be a finite von Neumann algebra, and let $\tau_{L}$ be a fixed normal faithful tracial state. Let $M=M_{n}(\mathbb{C}) \otimes L$, and let $\tau_{M}=\operatorname{Tr} / n \otimes \tau_{L}$. Let $u$ be a unitary in $M_{n}(\mathbb{C}) \otimes L$, and let $u=\sum_{i, j} e_{i j} \otimes u_{i j},\left(u_{i j} \in L\right)$, where $\left\{e_{i j}\right\}_{i, j=1, \cdots, n}$ is a set of matrix units of $M_{n}(\mathbb{C})$. We consider the set

$$
U=\left\{\frac{1}{\sqrt{n}} u_{i j} ; i, j=1, \cdots, n\right\} .
$$

It is not so essential, but we renumber the elements of $U$ for the sake of convenience. For example, if $k n+1 \leq i \leq(k+1) n$, for some $k=0,1, \cdots, n-1$, then we put

$$
u_{i}=\frac{1}{\sqrt{n}} u_{i-k n k+1} \text {. }
$$

It is clear the correspondence $i \longleftrightarrow(i-k n, k+1)$ for some $k=0,1, \cdots, n-1$ is one to one. Since $u$ is a unitary, clearly the set $U$ is a finite operational partition of unity of size $n^{2}$. We call this set $U$ the finite operational partition of unity induced by $u$. 
3.3.4. von Neumann entropy $S(\rho[U])$. We consider the von Neumann entropy $S\left(\rho_{\phi}[U]\right)$ of the density operator $\rho_{\phi}[U]$ in order to characterize the mutual orthogonality for subfactors. So, we assume that our state $\phi$ is the given normalized trace and

$$
S(\rho[U])=\operatorname{Tr}(\eta(\rho[U])) .
$$

3.3.5. Theorem. Let $L$ be a finite von Neumann algebra and let $\tau_{L}$ be a normalized trace of $L$. We let $M=M_{n}(\mathbb{C}) \otimes L$ and $\tau=\operatorname{Tr} / n \otimes \tau_{L}$. Assume that $N=$ $M_{n}(\mathbb{C}) \otimes 1_{L}$ and that $u$ is a unitary operator in $M$. Then the following conditions are equivalent:

(1) $N$ and $u N u^{*}$ are mutually orthogonal;

(2) $n^{2} \rho[U]$ is the $n^{2} \times n^{2}$ identity matrix;

(3) $S(\rho[U])=2 \log n=\log \operatorname{dim} N$.

Here $U$ is the finite operational partition of unity induced by $u$.

Proof. First we remark that

$$
\rho[U](i, j)=\frac{1}{n} \tau\left(u_{j-l n, l+1}^{*} u_{i-k n, k+1}\right)
$$

where $u_{i}=(1 / \sqrt{n}) u_{i-k n, k+1}$, for some $k=0,1, \cdots, n-1$ with $k n+1 \leq i \leq(k+1) n$, and $u_{j}=(1 / \sqrt{n}) u_{j-l n} l+1$, for some $l=0,1, \cdots, n-1$ with $l n+1 \leq j \leq(l k+1) n$.

(1) $\Rightarrow(2)$ : Assume that $N$ and $u N u^{*}$ are mutually orthogonal. Then by Theorem 3.2 .1 and by the definition of $\rho[U]$, the $n^{2} \times n^{2}$ density matrix $\rho[U]$ is the diagonal matrix such that

$$
\rho[U](i, i)=\frac{1}{n^{2}} \quad \text { for } i=1,2, \cdots, n^{2} .
$$

$(2) \Rightarrow(3)$ : Clearly, the the von Neumann entropy $S(\rho[U])=2 \log n$ and it is the dimension of $N$.

$(3) \Rightarrow(2)$ : Assume that $S(\rho[U])=\log n^{2}$. Let $\left(\lambda_{1}, \cdots, \lambda_{n^{2}}\right)$ be an eigenvalue sequence of $\rho[U]$ and let $\left(p_{1}, \cdots, p_{n^{2}}\right)$ be the corresponding sequence of the minimal projections. Then there exists a $n^{2} \times n^{2}$ unitary matrix $w$ so that

$$
w \rho[U] w^{*}=\sum_{i=1}^{n^{2}} \lambda_{i} p_{i} .
$$

Since

$$
\log n^{2}=S(\rho[U])=\sum_{i=1}^{n^{2}} \eta\left(\lambda_{i}\right),
$$

it implies that, by the concavity of the function $\eta$,

$$
\lambda_{i}=\frac{1}{n^{2}} \quad \text { for all } \quad i=1,2, \cdots, n^{2}
$$

so that

$$
w \rho[U] w^{*}=\frac{1}{n^{2}} 1_{M_{n^{2}}(\mathbb{C})} .
$$

Hence (2) holds. 
$(2) \Rightarrow(1)$ : By the definition of $\rho[U]$ and the condition (2), we have that

$$
\delta_{i j} \frac{1}{n^{2}}=\rho[U](i, j)=\frac{1}{n} \tau\left(u_{j-\ln l+1}^{*} u_{i-k n k+1}\right) .
$$

This relation corresponds that $\tau_{L}\left(u_{i j}^{*} u_{k l}\right)=\delta_{i k} \delta_{j l} \frac{1}{n}$. Hence by Theorem 3.2.1, $N$ and $u N u^{*}$ are mutually orthogonal.

3.3.6. Note. Theorem 3.3.5 means that the mutually orthogonality for inner conjugate subfactors are characterized by the maximum value log dim of the subfactors.

In fact, since the density matrix $\rho[U]$ is a $n^{2} \times n^{2}$ matrix and the function $\eta$ is operator concave, the value $2 \log n$ is the maximum.

3.3.7. Note. The proof shows that the statement of Theorem 3.3.5 does not depend on any choice of a matrix units.

3.4. Subfactors of matrix algebras. Let $A$ and $B$ be subalgebras of $M_{k}(\mathbb{C})$ and assume that both subalgebras are isomorphic to $M_{n}(\mathbb{C})$. Then $k=m n$. We can assume that $M_{k}(\mathbb{C})=M_{n}(\mathbb{C}) \otimes M_{m}(\mathbb{C})$ and $A=M_{n}(\mathbb{C}) \otimes \mathbb{C} 1$. There exists a unitary matrix $u \in M_{k}(\mathbb{C})$ such that $B=u A u^{*}$. We denote by $u(A, B)$ this unitary and also by $U(A, B)$ the finite operational partition of unity induced by $u(A, B)$. Then we have the followings:

3.4.1. Petz's characterization of complementarity was given in ([7, Theorem 4]): The subalgebra $u\left(1 \otimes M_{m}(\mathbb{C})\right) u^{*}$ is complementary to $1 \otimes M_{m}(\mathbb{C})$ if and only if

$$
\frac{m}{n} \sum_{i, j=1}^{n}\left|u_{i j}><u_{i j}\right|=1 .
$$

When $\mathrm{n}=\mathrm{m}$ this condition means that $\left\{u_{i j}\right\}_{i j}$ is an orthonormal basis in $M_{n}(\mathbb{C})$ with respect to the inner product by Tr.

Our characterization is the following Corollary of Theorem 3.3.6 by letting $L=$ $M_{m}(\mathbb{C})$.

3.4.2. Corollary. Let $A$ and $B$ be subalgebras of $M_{k}(\mathbb{C})$ and assume that both subalgebras are isomorphic to $M_{n}(\mathbb{C})$. Then $A$ and $B$ are mutually orthogonal if and only if

$$
S(\rho[U(A, B)])=2 \log n=\log (\operatorname{dim} A) .
$$

3.4.3. Note. In the above 3.4.1 and 3.4.2, the numbers $m$ and $n$ should be $m \geq n$.

3.4.4. Comparison with the case of maximal abelian subalgebras. We remark that Corollary 3.4.2 corresponds to [2, Corollary 3.2, Corollary 3.3]:

Assume that $A$ and $B$ are maximal abelian subalgebras of $M_{n}(\mathbb{C})$. Then there exists a unitary $u$ in $M_{n}(\mathbb{C})$ with $u A u^{*}=B$, and we have that

(1) $h(A \mid B)=H(b(u))$.

(2) $A$ and $B$ are mutually orthogonal if and only if

$$
h(A \mid B)=\log n=\log (\operatorname{dim} A) .
$$


Here, $h(A \mid B)$ is the conditional relative entropy for $A$ and $B$ in [2] and $H(b(u))$ is the entropy for the unistochastic operator $b(u)$ induced by the unitary $u$ in [11].

This means that $A$ and $B$ are mutually orthogonal if and only if $h(A \mid B)$ takes the maximum value $\log (\operatorname{dim} A)$, because $\log n$ is the maximum value by the definition of $H(b(u))$ and by the property of the function $\eta$.

\section{REFERENCES}

1. R. Alicki and M. Fannes, Defining Quantum Dynamical entropy, Lett. Math. Phys., 32 (1994), $75-82$.

2. M. Choda, Relative entropy for maximal abelian subalgebras of matrices and the entropy of unistochastic matrices, Internat. J. Math., 19(2008), no. 7, 767-776.

3. M. Choda, Conjugate Pairs of Subfactors and Entropy for Automorphisms, arXiv:1002.0631, to appear in Internat. J. Math..

4. A. Connes and E. Størmer, Entropy of $I I_{1}$ von Neumann algebras, Acta Math., 134 (1975), 289-306.

5. S. Neshveyev and E. Størmer, Dynamical entropy in operator algebras, Springer-Verlag, Berlin (2006).

6. M. Ohya and D. Petz, Quantum entropy and its use, Texts and Monographs in Physics. Springer-Verlag, Berlin (1993).

7. D. Petz, Complementarity in Quantum Systems, Reports Math. Physics 59(2007), 209 -224.

8. D. Petz, Algebraic complementarity in quantum theory, J. Math. Phys. 51 (2010),

9. D. Petz, A. Szántó and M. Weiner, Complementarity and the algebraic structure of 4-level quantum systems, Infin. Dimens. Anal. Quantum Probab. Relat. Top., 12 (2009), 99-116.

10. S. Popa, Orthogonal pairs of *-subalgebras in finite von Neumann algebras, J. Operator Theory, 9 (1983), 253-268.

11. K. Życzkowski, M. Kuś, W. Słomczyński and H.-J. Sommers, Random unistochastic matrices, J. Phys., A36 (2003), 3425-3450. 